\title{
La chirurgie de l'appareil génital mâle à Byzance
}

\author{
Georges ANDROUTSOS
}

Histoire de la Médecine, Faculté de Médecine, Université d'loannina, Grèce

\begin{abstract}
RESUME
Les opérations qui étaient effectuées sur les testicules et le scrotum à l'époque byzantine montrent les conditions excellentes d'exercice et l'évolution considérable de la chirurgie urologique à Byzance ainsi que le niveau élevé de la chirurgie byzantine en général.
\end{abstract}

Mots clés : Byzance, chirurgie, testicules, scrotum

\section{INTRODUCTION}

Les interventions sur les testicules et le scrotum constituaient une partie importante de la chirurgie urologique byzantine et leur pratique courante dans les hôpitaux de l'empire a contribué à l'invention de nouvelles techniques et à leur amélioration. Le traitement chirurgical de l'hydrocèle grâce à l'utilisation de drainage par contre-ouverture, la castration, le traitement chirurgical de la varicocèle et des fistules scrotales ainsi que la réparation pour des raisons esthétiques de la laxité du scrotum, constituent les interventions les plus importantes parmi celles que décrivent les textes historiques byzantins. Leur mention par de nombreux écrivains byzantins et les diverses techniques qui sont décrites, notamment l'emploi alternatif du bistouri et des cautères, même les efforts ratés de remplacement des opérations chirurgicales, soulignent la continuité diachronique de la chirurgie urologique et manifestent le niveau élevé de la chirurgie byzantine [6].

\section{GENERALITES}

L'époque byzantine est reconnue à l'heure actuelle comme une période de prospérité et de floraison de la médecine malgré les opinions contraires des historiens plus anciens. Ceci est vrai, notamment pour la chirurgie où des inter- ventions de types, de tailles et de gravités diverses étaient effectuées dans les hôpitaux byzantins, qui représentent les premières institutions purement hospitalières dans I'histoire qui disposaient d'un service médical et infirmier organisé et hiérarchisé [7]. A titre indicatif nous mentionnons l'opération de séparation de deux jumeaux siamois

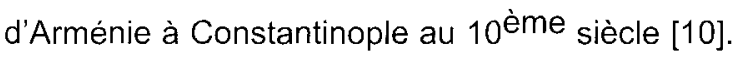

Dans cet article nous traitons des interventions sur le scrotum et les testicules.

\section{HYDROCELE}

Le traitement chirurgical de l'hydrocèle était une des interventions les plus courantes effectuées à Byzance [3]. Sa description devait exister dans l'œuvre d'Oribase (325403), médecin célèbre et ami de l'empereur byzantin Julien dit l'Apostat (331-363), étant donné les références contenues à ce sujet dans d'autres chapitres, mais les passages correspondants n'ont pas été préservés [8]. L'opération est citée par Aetius d'Amide (502-575), médecin de l'empereur Justinien (482-565) [4], et par Léon l'latrosophiste, médecin célèbre du 10 ème siècle [5]. La description détaillée, cependant, a été faite par Paul d'Egine (625-690), médecin byzantin éminent, dernier grand représentant de l'Ecole de Médecine d'Alexandrie.

Paul d'Egine décrit deux opérations en utilisant respectivement le bistouri et les cautères.

\section{Opération au bistouri [9] (Figure 1)}

Après avoir rasé les poils du scrotum et du pubis, on met le patient sur le dos. Le chirurgien est debout à gauche du patient tandis que son assistant refoule la verge et la peau du scrotum vers l'abdomen. L'incision cutanée scrotale est

\section{Correspondance :}

Dr Georges ANDROUTSOS, 1 rue Ipeirou, 10433, Athènes, Grèce - Email lyon 48@otenet.gr 


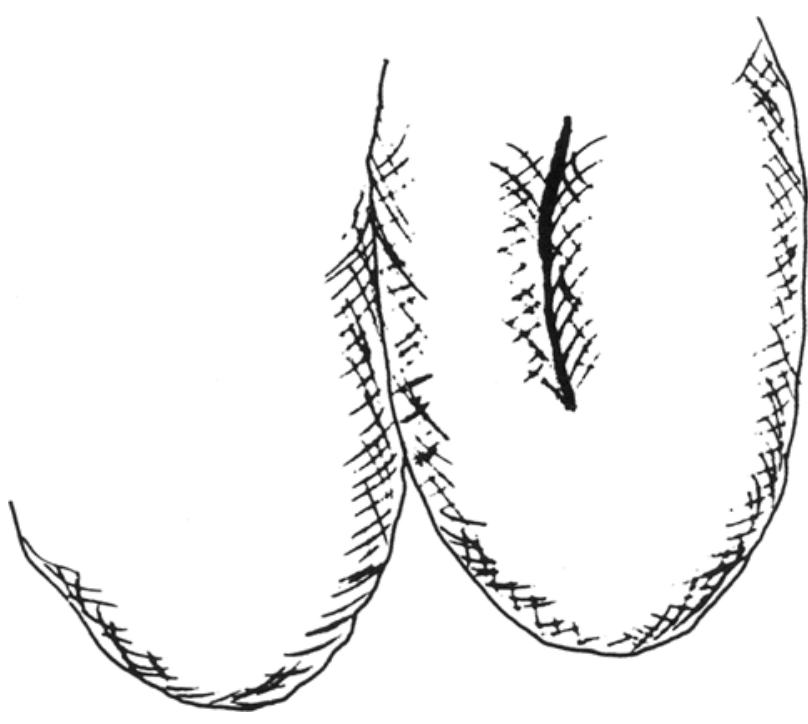

Figure 1 : Représentation schématique de l'incision de l'opération de l'hydrocèle selon Paul d'Egine.

verticale, parallèle au raphé du scrotum depuis le milieu jusqu'au pubis et traverse les plans cutanés, le dartos, et la fibreuse jusqu'au reflet bleuté de la vaginale qui se décolle à l'aide d'un instrument spécial, le « couteau à hydrocèle » et, après l'avoir disséquée, on l'entrecoupe au milieu et au point le plus distant du testicule. Après l'évacuation du liquide pathologique, la vaginale est soutenue par des crochets et réséquée d'un seul tenant, soit après fente de la séreuse en deux points opposés (Figure 2). En fin d'intervention, les plus anciens procédaient à la suture de la plaie. Paul d'Egine préfère la fermeture pariétale en deuxième but. A travers l'incision, on introduit un stylet cannelé jusqu'au sommet du scrotum et sur cette cannelure longitudinale on fait l'incision "sous écoulement ", c'est-à-dire une contreouverture « pour que les caillots et le pus soient évacués ». Le drainage est effectué au moyen d'une gaze triangulaire qui sort à travers une contre-ouverture et qui est introduite à l'aide du stylet par l'incision principale. L'ablation de la gaze s'effectue le 7 ème jour post-opératoire (Figure 3). On place deux pansements superposés sur la plaie : une laine imprégnée d'huile à l'intérieur, et une laine imprégnée d'huile et vin à l'extérieur. On finit par un bandage compressif.

Après l'opération, le patient reste sur le dos tandis que la plaie est arrosée avec de l'huile tiède. Le 3ème jour, on renouvelle le pansement et le 7ème our, on procède à l'ablation du pansement et le patient peut se laver.

Il est évident que les premiers temps opératoires sont restés les mêmes jusqu'à aujourd'hui, mais aussi les autres temps opératoires, même différents aujourd'hui, visent au même but, la formation de cicatrice qui fera disparaître la cavité de la vaginale. La mise en place d'un drainage à travers une contre-ouverture, identique à celui d'aujourd'hui, montre la grande expérience des médecins byzantins sur

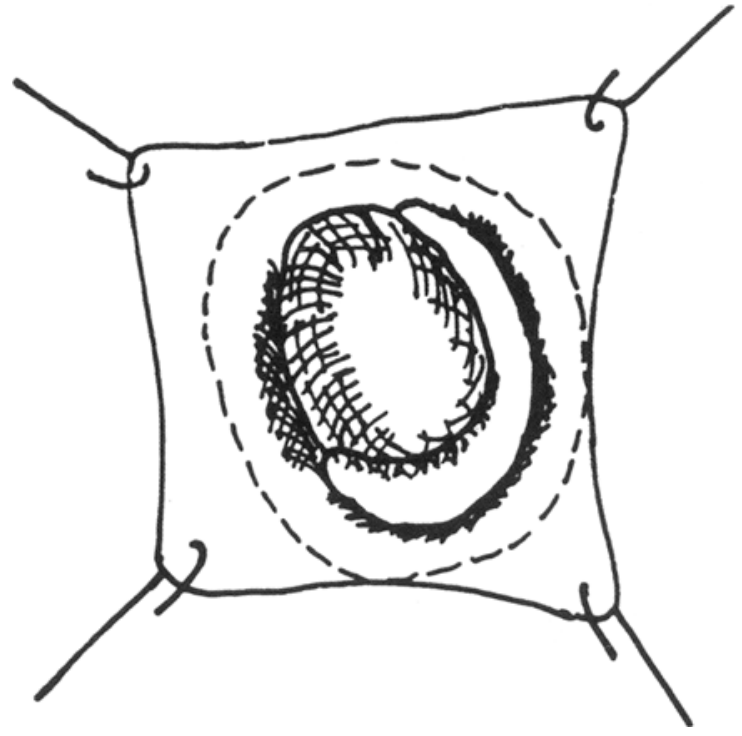

Figure 2 : Représentation schématique de l'incision de l'opération de l'hydrocèle selon Paul d'Egine. On discerne le même tissu vaginal qui a été creusé et qui est retenu par des crochets tandis que la ligne circulaire pointillée représente la ligne de détachement.

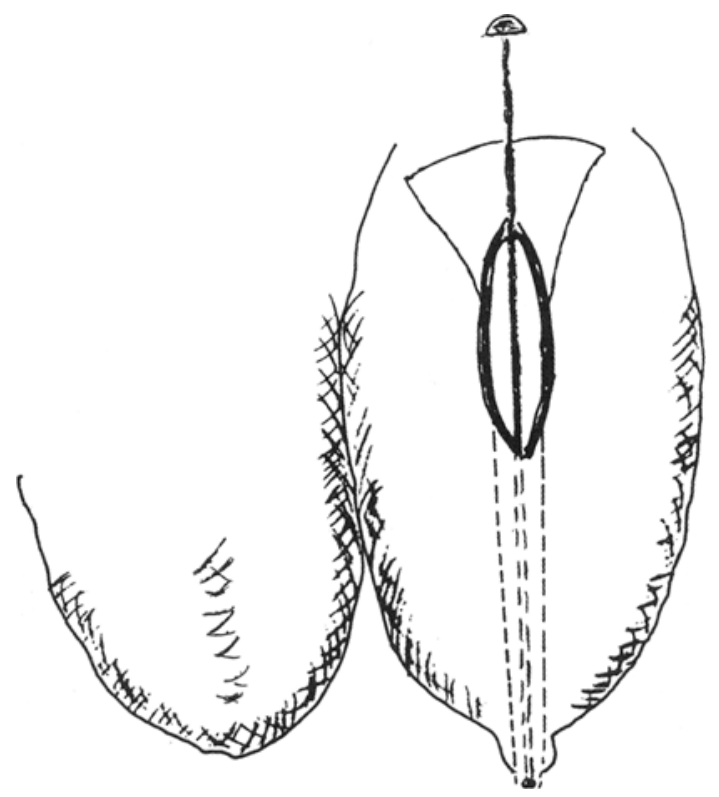

Figure 3 : L'incision " sous écoulement ". On note le stylet introduit par l'incision principale qui fait saillir la peau par-dessous au point de la contre-ouverture mais aussi le " Jemnisque " qui s'est rendu au drainage.

l'opération et par conséquent la connaissance de l'impossible hémostase complète du scrotum, ce qui reste en vigueur jusqu'à présent.

\section{Opération aux cautères}

Paul d'Egine (PAUL D'EGINE, op. cit., 268), bien qu'il décrit 
en détail l'opération au bistouri, il semble qu'il préfère le procédé opératoire aux cautères. On utilise deux types de cautères, les " linéaires " à la forme de $\Gamma$ (lettre grecque majuscule) qui sont larges pour les parois extérieures et les " types en couteau " qui sont plus fins et aigus pour les parois intérieures. Les cautères sont rougis au feu et l'opération se réalise avec la cautérisation graduelle des plans du scrotum après les avoir disséqués selon le procédé décrit ci-dessus jusqu'à la vaginale qui est disséquée et incisée avec la pointe du cautère linéaire. Après l'évacuation du liquide pathologique, la vaginale se décolle en cycle avec les cautères en forme de couteau. On ne procède pas à un drainage à travers une contre-ouverture car l'hémostase aux cautères est considérée comme satisfaisante. Les autres temps et les soins post-opératoires sont pareils aux temps respectifs de l'opération conventionnelle.

\section{Usage des substances caustiques}

Aetius d'Amide, bien qu'il se réfère à l'opération aux cautères, suggère une méthode différente pour le traitement de l'hydrocèle au moyen des substances caustiques, comme la térébenthine et la " mousse d'argent», probablement poudre de nitrate d'argent (FROBEN, op. cit., XIV : 20) en provoquant une brûlure chimique au lieu de la brûlure thermique des cautères. Selon cette méthode, on enduit le scrotum avec les substances caustiques en étendue analogue au volume de l'hydrocèle. La brûlure chimique résultée, pénètre les enveloppes du scrotum jusqu'à la vaginale d'où le contenu de l'hydrocèle est évacué. Suit l'application locale des substances caustiques dans la cavité de la vaginale afin de faire une cicatrice et fermer la cavité pour éviter la récidive de l'hydrocèle.

Cette méthode n'est mentionnée par aucun autre écrivain médical byzantin, plutôt parce qu'elle n'était pas efficace.

\section{CASTRATION}

La castration (orchidectomie, orchiéctomie) à l'époque byzantine était pratiquée pour des raisons thérapeutiques et pour créer des eunuques [1]. L'opération est mentionnée par Oribase (ORIBASE, op. cit., $3: 634$ ) et Paul d'Egine (PAUL D'EGINE., op. cit., 64).

L'opération commence de la même façon que l'opération de l'hydrocèle jusqu'à l'ouverture de la vaginale. Ensuite, on dissèque le testicule, on divise les vaisseaux qui sont ligaturés et sectionnés du déférent qui est juste sectionné et le testicule est enlevé en bloc en tant que " corps étranger". En ce moment, on draine le scrotum au moyen d'une gaze triangulaire qui sort à travers une contre-ouverture. Si le testicule est adhéré à ses enveloppes, on enlève tout le testicule en bloc sans dissection [2]. Comme il résulte, l'opération était pareille à l'actuelle.

\section{VARICOCÈLE}

L'opération de la varicocèle remonte aux byzantins. On trouve la plus ancienne référence dans les écrits d'Oribase (ORIBASE, op. cit., $4: 44$ ) tandis que Paul d'Egine (PAUL D'EGINE., op. cit., 272) décrit en détail sa propre technique.
En plus, l'opération est aussi citée par Léon l'latrosophiste [5] et Aetius d'Amide (FROBEN, op. cit., 68).

Oribase pratique des incisions multiples le long des veines variqueuses à intervalles d'un pouce avec des ligatures respectives ou cautérisation des veines (Figure 4). Par contre, Paul d'Egine fait juste une incision après avoir refoulé vers le bas le canal déférent avec les doigts. L'incision se fait au-dessus des vaisseaux qui sont tenus par le chirurgien et l'assistant. Après la dissection des vaisseaux, on passe par dessous de chaque vaisseau une aiguille au double fil de lin. Le fil est coupé au bout et donc on a deux fils avec lesquels est ligaturé le vaisseau en amont et en aval de la dilatation variqueuse. Après la ligature, les vaisseaux sont sectionnés et enlevés. La plaie reste ouverte et pansée jusqu'à sa cicatrisation en deuxième but et la chute des sutures. L'opération de Paul d'Egine, de la ligature des vaisseaux au niveau du scrotum (Figure 5), pratiquée jusqu'aux années 60 , a cédé sa place, progressivement, au procédé opératoire proposé par De Palomo, de la ligature haute de la veine spermatique.

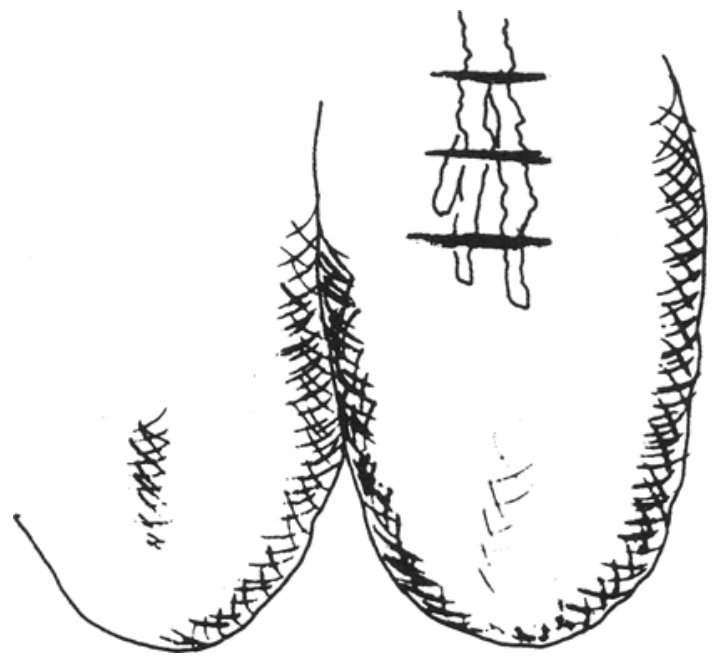

Figure 4 : L'opération de la varicocèle selon Oribase.

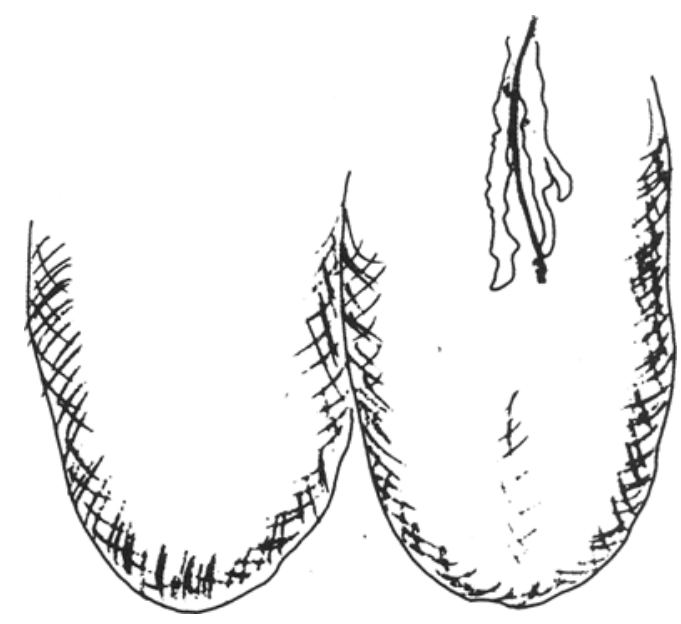

Figure 5 : L'opération de la varicocèle selon Paul d'Egine. 


\section{FISTULES DU SCROTUM}

Les fistules de scrotum sont mentionnées par Oribase (ORIBASE, op. cit., $3: 633$ ) et classées en antérieures, qui pénètrent la vaginale et postérieures, qui arrivent jusqu'au mesorchium (mésotestis). Quand on introduit un stylet à une fistule antérieure, on sent qu'il est vide avant de toucher le testicule et que le patient ait mal, tandis que ce dernier a immédiatement mal lors de l'exploration des fistules postérieures (Figure 6).

Le traitement consiste à l'excision de la fistule et on recommande l'incision le long du stylet et l'excision du canal fistuleux. Si le testicule est atteint, est indiquée la castration.

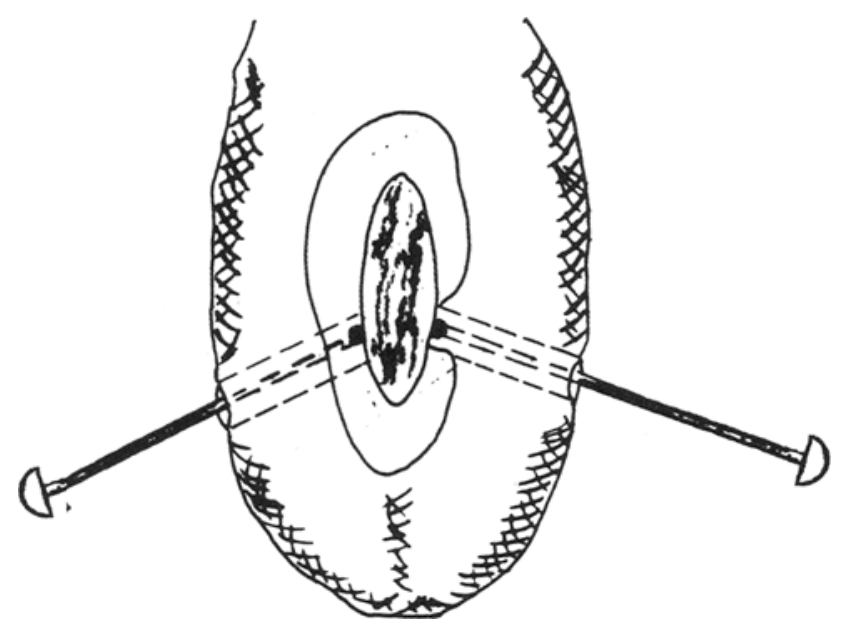

Figure 6 : Le sondage (exploration par un stylet) des fistules du scrotum.

\section{LAXITE SCROTALE (SCROTUM FLASQUE)}

L'excessive laxité scrotale et le désir de constituer un suspensoir naturel constituaient chez les Byzantins une indication rare mais légitime d'amélioration esthétique et parfois de suppression d'une tension douloureuse. Paul d'Egine mentionne cette pathologie en tant que "scrotum plissé» et pour sa réparation, on procède à l'unique, avec la reconstitution du prépuce défectueux, opération urologique réparatrice des temps byzantins.

L'opération consiste à la résection de l'excès de peau scrotale et a deux variantes. Selon la première, on place le scrotum sur un support en bois ou en cuir et la peau en excès est réséquée. En fin d'intervention on procède à la fermeture de la plaie par une suture cutanée.

La deuxième variante utilise un fil tracteur cutané au-dessous de l'angle péno-scrotal, un second fil proche du périnée et un troisième au sommet de l'excès de peau, de telle sorte que le sacrifice utile montre clairement ses limites (Figure 7).

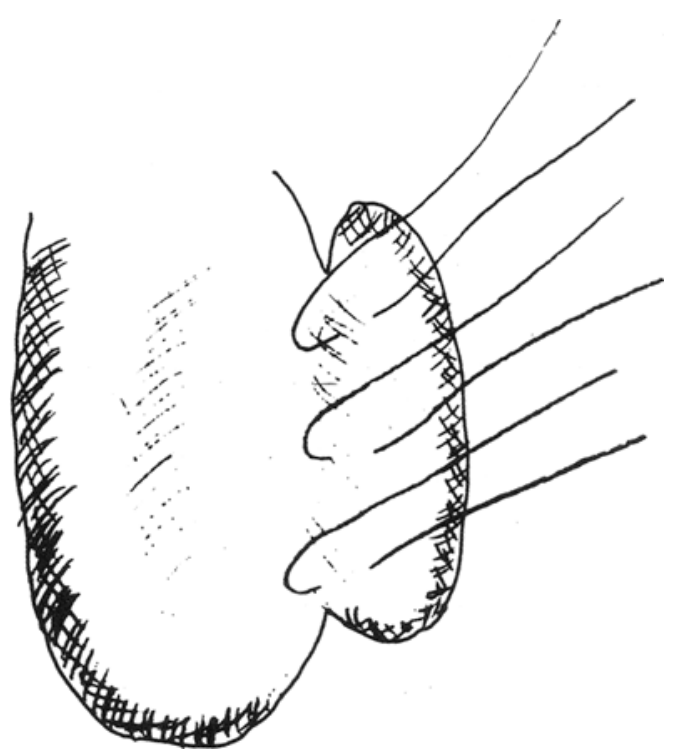

Figure 7 : La correction chirurgicale du scrotum flasque.

\section{DISCUSSION}

Les descriptions de ces opérations mentionnées dans plusieurs oeuvres byzantines, nous montrent qu'à l'époque byzantine, elles étaient très courantes, ce qui résultait à l'amélioration continue des techniques opératoires. La dissection des veines variqueuses de la varicocèle par Paul d'Egine, au 6ème siècle, au lieu des multiples incisions aveugles d'Oribase, au 4ème siècle, est un exemple caractéristique. D'ailleurs, l'usage de drainage du scrotum à travers une contre-ouverture montre la bonne connaissance et la longue expérience des médecins byzantins à ce genre d'interventions. Même les tentatives les plus ratées, comme l'usage des substances caustiques par Aetius d'Amide pour le traitement de l'hydrocèle, montre l'esprit clinique des médecins byzantins, résultat de leur expérience clinique.

Par conséquent, on peut constater que les opérations aux testicules et au scrotum pendant l'époque byzantine montrent la familiarisation des médecins avec l'objet à travers la pratique fréquente et incessante des opérations urologiques chirurgicales, résultat du niveau supérieur de la médecine byzantine.

\section{REFERENCES}

1. ANDROUTSOS G., MARKETOS S.P. : La castration à travers les âges. Andrologie, 1993, $3: 61-66$.

2. ANDROUTSOS G., GEROULANOS S. : L'eunuchisme à Byzance. Progrès en Urologie, 2001, 11 : 757-760.

3. ANDROUTSOS $\mathrm{G}$. : Les divers types d'eunuques de l'État Byzantin : leur rôle et leur sens symbolique. Sexologies, 2003, $12: 39-50$.

4. FROBEN : Aeti Amideni Libri XII, Basileae, 1535, Liber XIV : 20. 
5. LEON L'IATROSOPHISTE : Synopsis latrikis. Ermerins FZ (ed). Anecdota Medica Graeca, Lugduni Batavorum (Leyden), Luchtmans, 1840, 80-221: 197.

6. MALAKATES $S$. : Scrotal and testicular operations in the Byzantium. Hellenic Urology, 1997, 2 : 212-217.

7. MILLER T.S. : The Birth of the Hospital in the Byzantine Empire. Baltimore and London, The John Hopkins University Press, $1985: 1-11$.

8. ORIBASE : CEuvres d'Oribase. Trad. Darenberg. Paris, Busemaker Ed. Molinier,1851-1876, 4 : 495.

9. PAUL D'EGINE : Chirurgie. Trad. R. Briau, Paris, 1855.

10. PENTOGALOS G., LASCARATOS J. : A surgical operation performed on Siamese twins during the tenth century in the Byzantium. Bull. Hist. Med., 1984, 58 : 99-102.

Manuscrit reçu : février 2005 ; accepté février 2005.

\author{
ABSTRACT \\ Male genital tract surgery in Byzantium
}

Georges ANDROUTSOS

Medicine and surgery developed and flourished in the Byzantine age, despite the opposite opinions of earlier historians. Operations on the testicles and scrotum constituted an important part of Byzantine urological surgery and were regularly performed in Byzantine hospitals, which constituted a radically new innovation for the development and improvement of operative techniques.

Surgical repair of hydrocele with the all-important addition of drainage through a separate lower incision, orchidectomy, surgical resection of varicocele and scrotal fistulae as well as repair of the loosened scrotum for aesthetic reasons are the most commonly described operations in this anatomical region in Byzantine medical texts.

The descriptions by many authors, from the fourth to the eleventh century A.D., and the various techniques described, especially alternation of scalpel and cautery, indicated the continuous use of these operations and the practice of surgery and medicine in general, during the centuries of the Byzantine age.

In conclusion, operations on the testicles and scrotum performed in the Byzantine age demonstrate the excellent conditions of medical practice and the development of urological surgery in Byzantium and the high level of Byzantine surgery and medicine.

Key-words : Byzantium, surgery, testicles, scrotum 